\title{
ESTRATEGIAS PARA LA DOBLE CAMPAÑA DE PRODUCCIÓN EN LA ZONA ANDINA DEL PERÚ
}

\author{
STRATEGIES FOR THE DOUBLE PRODUCTION CAMPAIGN IN THE \\ ANDEAN ZONE OF PERU \\ (iD) Juan Quispe Rodríguez ${ }^{*}$, (iD Uriel Rigoberto Quispe Quezada1, (iD José Antonio Quispe \\ Medrano $^{2}$
}

jquisper@unah.edu.pe; uquispe@unah.edu.pe; jhoseph@gmail.com

${ }^{1}$ Universidad Nacional Autónoma de Huanta, Ayacucho, Perú

${ }^{2}$ Consultor de la empresa "Xperis" Manpower group

*Correspondencia: Juan Quispe Rodríguez. Email: jquisper@unah.edu.pe

Recibido: 04.03.2020 | Aprobado: 09.04.2020

\begin{abstract}
RESUMEN
La doble campaña de producción en la agricultura, dicho de otra manera, es obtener dos cosechas en un año, la misma que es importante considerar en una agricultura eficiente y de buena rentabilidad, por lo mismo que es importante que el sector agroalimentario deberá adecuarse al ritmo de la competitividad necesaria a fin de convertirse en verdaderos centros de producción orientados al mercado con precios justos, mejorando los modelos de gestión, control de los procesos que faciliten la toma de decisiones e incorporen los avances tecnológicos; en muchos lugares se ha realizado proyectos de irrigación que facilitan el uso y manejo adecuado del agua de riego, sin embargo en muchas de ellas no consideran un aspecto muy importante como es el "desarrollo agrícola" que es la actividad decisiva para evaluar el impacto del proyecto y que a la larga será la que justifique la inversión realizada cuando los agricultores mejoren sus ingresos por mejor organización, aplicación de tecnologías, capacidad de negociación y mayor rentabilidad de sus productos, y consecuentemente mejoren su nivel y calidad de vida. En el presente documento se hace conocer las labores de investigación que se realizaron en el valle del Mantaro y otras zonas de la región Junín - Perú, con buenos resultados considerando cultivos tolerantes a las bajas temperaturas, precoces y de buena rentabilidad, uso eficiente del agua de riego y se propone además una cedula de cultivos base que se puede considerar para otras zonas interandinas.

Palabras clave: Doble campaña, cultivos, zona interandina, tecnologías, bajas temperaturas, heladas.
\end{abstract}

\begin{abstract}
The double production campaign in agriculture, in other words, is to obtain two crops in one year, which is important to consider in an efficient and profitable agriculture, so 1 it is important that the agri-food sector must adapt to the pace of the necessary competitiveness in order to become true centers of production oriented to the market with fair prices, improving the management models, control of the processes that facilitate the decision making and incorporate the technological advances; In many places irrigation projects have been carried out that facilitate the use and proper management of irrigation water, however in many of them they do not consider a very important aspect such as "agricultural development" which is the decisive activity to assess the impact of project and that in the long run it will be the one that justifies the investment made when farmers improve their income by better organization, application of technologies, negotiation capacity and greater profitability of their products and consequently improve their level and quality of life. This document makes known the research work carried out in the Mantaro Valley and other areas of the Junín - Peru region. with good results considering tolerant crops at low temperatures, early, and of good profitability, efficient use of irrigation water and also proposes a basic crop card that can be considered for other interAndean areas.
\end{abstract}

Keywords: Double campaign, crops, interandina zone, technologies, low temperatures, frost. 


\section{INTRODUCCIÓN}

En el Perú se han realizado numerosas obras de irrigación por instituciones como el Proyecto especial de pequeñas y medianas irrigaciones PEPMI, el Instituto Nacional de Ampliación de la Frontera Agrícola INAF, en la actualidad con otros nombres como la Administración Nacional de Agua ANA, a nivel nacional y la Administración Local del Agua ALA. A nivel local, que después de la construcción de la infraestructura mayor de riego (bocatoma, canal principal y secundario, obras de arte, etc.) era complementada con las acciones de "Desarrollo Agrícola" (1994) la que prácticamente justificaba la inversión realizada por el estado peruano al obtener mayor producción y productividad agrícola y pecuaria así como elevar las condiciones de vida de los agricultores, utilizando en forma eficiente los recursos tierra y agua durante los 12 meses del año con desarrollo de programas de investigación y demostración a los agricultores, usuarios de los proyectos de riego.

A la fecha se continúan construyendo infraestructuras de riego mayor sin considerar las acciones de desarrollo agrícola (considerándose en ella la doble campaña de producción), por lo que en muchos casos los usuarios de regantes organizados en comisión y comités de regantes necesitan capacitación y adiestramiento en el mejor uso y manejo del agua de riego con implementación de un riego tecnificado a fin de elevar la eficiencia en por lo menos a un 80 o $90 \%$ superando lo que actualmente se tiene con riego por superficie en solo un 35\% de eficiencia perdiéndose el 65\% ya sea por escorrentía, percolación profunda y evaporación, básicamente ocurre esto por la poca pericia que tienen la mayoría de agricultores en el manejo del agua de riego.

En esta actividad uno de los factores importantes es la consideración de variables climáticas, cuyos registros continuos y sistemáticos permitirán determinar la evolución en el tiempo de las variables de mayor importancia, los datos meteorológicos cada vez son más indispensables para poder contar con predicciones más precisas, El comportamiento del clima está sujeto a muchas variables. La temperatura junto a la humedad relativa, son las variables climáticas más importantes. Incluso la temperatura es una variable básica para establecer las clasificaciones climáticas a cualquier escala (MOPT, 1992).

El conocimiento del requerimiento hídrico de los cultivos es de gran importancia para la planificación de cultivos y programación de riegos, así como de la operación de los proyectos de riego, las mismas que existen tanto a nivel local, regional, nacional y mundial. El uso del agua (captación y consumo) por los diferentes sectores se basa generalmente en estimaciones y no en medidas reales, la agricultura representa aproximadamente el $70 \%$ de la captación total de agua dulce a nivel mundial y más del 90\% en la mayoría de los países menos desarrollados (Naciones Unidas Para la Agricultura y la Alimentación FAO, 2011a).

Del mismo modo en estas zonas agrícolas, las prácticas de riego se realizan de acuerdo con la experiencia solo del agricultor con base en la aplicación uniforme de riego durante todo el ciclo vegetativo del cultivo, sin considerar las exigencias o demanda hídrica del cultivo y las condiciones climáticas en un momento determinado, por ello es necesario aplicar los riegos considerando la necesidad hídrica del cultivo. En la zona de sierra o zona andina, no existe información de ETP útil para la planificación agrícola, 
balances hídricos, demanda de agua de los cultivos, programa de riego y eficiencia de riego (Garay, 2009)

El Instituto Nacional de Estadística e Informática INEI según el IV censo nacional agropecuario nos dice que el Perú cuenta con 7125007 has. cultivables, de las cuales 4 545107,9 has. son áreas en secano (62.8\%) y 2579899,9 has. son áreas bajo riego (36,2\%) de las cuales el 51\% del área total se encuentran en la zona de sierra (Instituto Nacional de Estadística e Informática INEI, 2012)

De todo ello uno de los aspectos más importantes a considerar en la planificación de la doble campaña de producción es la variable temperatura, sobre todo las medias y la mínimas, Es de todos conocido que la temperatura es una de las magnitudes más utilizadas para describir el estado de la atmósfera, de hecho, la información meteorológica que aparece en los medios de comunicación casi siempre incluye un apartado dedicado a las temperaturas: sabemos que la temperatura del aire varía entre el día y la noche, entre una estación y otra, y también entre una ubicación geográfica y otra. En invierno puede llegar a estar bajo los $0^{\circ} \mathrm{C}$ y en verano superar los $40^{\circ} \mathrm{C}$. (Rodríguez et al., 2004)

Considerando toda esta información es que se planifica las estrategias para realizar con éxito una doble campaña de producción sobre todo en la zona andina del Perú.

\section{METODOLOGÍA}

Los estudios se basan principalmente en la obtención de respuestas a ciertos factores climáticos de los cultivos para la obtención de la doble campaña de producción anual, frecuencias de riego y determinación de coeficientes hídricos para algunos cultivos de sierra, este último muy importante para determinar la demanda de agua de los cultivos propuestos.

\section{Situación, fisiografía y clima}

La estación experimental se encuentra situada en el distrito de el Tambo a $3.5 \mathrm{~km}$. de la ciudad de Huancayo, en la margen izquierda del rio Mantaro, sobre una terraza aluvial media, el clima predominante se define por la concurrencia de lluvias entre los meses de octubre a abril y de sequias el resto del año, así como por temperaturas bajas (heladas) durante los meses de mayo a julio.

\section{Tolerancia de cultivos a bajas temperaturas.}

El efecto de las bajas temperaturas sobre los cultivos es función de las temperaturas medias mínimas, las mismas que dependen de la intensidad y duración sobre la superficie del terreno, así como del estado fisiológico de la planta para ocasionar daño. Sin embargo, existen cultivos y variedades que muestran tolerancia e inclusive resistencia a las condiciones adversas de las bajas temperaturas, manifestándose estas en el tamaño de la planta y fruto, alargamiento del periodo vegetativo, germinación y/o brotamiento.

Así mismo las temperaturas diarias, registradas en las estaciones meteorológicas no siempre son las mismas temperaturas que actúan sobre las plantas, en el mismo cuadro 1 se aprecian las temperaturas mínimas, máximas y media mensuales, durante los años de registro, registrados en la misma estación experimental de Santa Ana del INIA, en la ciudad de Huancayo. 


\section{Cuadro 1}

Temperaturas máximas, medias y minimas (2000-2010)

\begin{tabular}{lcccccccccccc}
\hline Dato & $\mathbf{E}$ & $\mathbf{F}$ & $\mathbf{M}$ & $\mathbf{A}$ & $\mathbf{M}$ & $\mathbf{J}$ & $\mathbf{J}$ & $\mathbf{A}$ & $\mathbf{S}$ & $\mathbf{O}$ & $\mathbf{N}$ & $\mathbf{D}$ \\
\hline $\begin{array}{l}\text { Temp. } \\
\text { Max. }\end{array}$ & 19.5 & 19.2 & 18.7 & 19.8 & 20.9 & 20.4 & 20.2 & 20.5 & 20.4 & 20.7 & 21.2 & 20.0 \\
$\begin{array}{l}\text { Temp. } \\
\text { Med. }\end{array}$ & 12.9 & 12.8 & 12.4 & 12.2 & 11.8 & 10.9 & 10.7 & 11.7 & 12.6 & 13.3 & 13.7 & 13.3 \\
$\begin{array}{l}\text { Temp. } \\
\text { Min. }\end{array}$ & 6.7 & 6.8 & 6.4 & 4.3 & 2.1 & 0.3 & 0.0 & 1.3 & 3.8 & 5.3 & 5.2 & 6.4 \\
\hline
\end{tabular}

Fuente: SENAMHI

De acuerdo a los datos registrados por la FAO, UNESCO, OMM (1975) en la zona andina, sobre temperaturas medias optimas y medias mínimas requeridas para el normal desarrollo de los cultivos, esta se ha relacionado con los registrados en la estación experimental Santa Ana para los cultivos en estudio como son:

\section{Papa}

Las respuestas fisiológicas de la papa a radiación, temperaturas bajas son muy variables, existen algunas especies de papas muy amargas tolerantes al frio, las mismas que se siembran a más de 3,500 msnm. Como en las localidades de Chicche y Yanacancha en Junín - Perú

El estudio de la FAO, UNESCO, OMM., (1975) indican que en Colombia algunas variedades mejoradas han resistido hasta $-2^{\circ} \mathrm{C}$. y una variedad hasta $-5^{\circ} \mathrm{C}$. con la temperatura medida a unos $15 \mathrm{~cm}$. Sobre el suelo.

\section{Haba}

Las habas suportan temperaturas bastante bajas y toleran las heladas, en las siembras que se realizaron en los meses de mayo a junio con temperaturas de $-3^{\circ} \mathrm{C}$ y hasta los $4^{\circ} \mathrm{C}$, observándose tolerancia a dichas temperaturas solo con algunas hojas afectadas que no hicieron daño significativo pues rápidamente se recuperaron, la época más crítica para las heladas es en la floración y el envainamiento por cuanto pueden producir una baja fecundación y formación de granos.

\section{Arveja}

El cultivo de la arveja igualmente soporta las bajas temperaturas y toleran las heladas, cuando las siembras se realizaron en los meses de mayo y junio no se observaron problema alguno significativo en su normal desarrollo, pero si con el inconveniente que este cultivo es muy susceptible a la humedad excesiva, por lo tanto los riegos se deben efectuar en forma ligera dependiendo de la textura de suelo.

\section{Trigo}

El cultivo del trigo necesita aproximadamente 1050 horas de sol para completar su ciclo vegetativo, cuando hay déficit de horas-sol, el periodo vegetativo se alarga significativamente, así como en la provisión de humedad constante, el periodo crítico para las heladas se observa que es durante el espigado y floración (Salazar, 1975) 
Este cultivo cesa de crecer completamente a los 5 a 6 grados centígrados (cero de su escala biológica), en las siembras realizadas en los meses de junio y julio con temperaturas de $0^{\circ} \mathrm{C}$ a $-4^{\circ} \mathrm{C}$. detuvieron su crecimiento en las primeras etapas de su desarrollo, a medida que avanzaba el tiempo y la subida de la temperatura recuperaban su vigor de crecimiento llegando a su normal desarrollo, pero con aumento en su ciclo vegetativo.

\section{Cebada}

La cebada de altura, por su precocidad en sus periodos de espigado a floración y formación de granos permiten utilizarlos fácilmente como uno de los cultivos de la doble campaña de producción durante las épocas de heladas, cuidando que los periodos críticos no caigan en los meses de mayor incidencia de heladas, en los altiplanos del Perú y Bolivia se utilizan mucho para forraje en vista que las condiciones de heladas y sequias, los ciclos vegetativos no llegan a producir calidad de grano aceptable.

\section{Hortalizas}

En el caso de las hortalizas como la col, lechuga, acelga, espinaca, cebolla, apio y zanahoria, fácilmente se pueden utilizar en época de heladas en terrenos bajo riego por cuanto muestran tolerancia a las bajas temperaturas y heladas, desarrollándose en forma normal con la salvedad que aumentan su periodo vegetativo en unos 15 a 30 días más a lo normal

\section{Temperaturas en la zona del valle del Mantaro}

En el cuadro 2, podemos apreciar las frecuencias e intensidad de heladas en la estación meteorológica de Huayao en Junín - Perú a 3,313msnm, en la cual se indican el número de años en que la temperatura llego a un registro menor o igual al indicado y los números en paréntesis $O$ indican el número total de observaciones en que llego la temperatura a ser menor o igual a lo indicado.

De acuerdo a este cuadro, se puede programar las fechas de siembra, así como el cultivo apropiado, teniendo en cuenta que no llegan a coincidir la floración hasta los inicios del mes de setiembre.

\section{Cuadro 2}

Frecuencia de temperaturas minimas, IGP. Huayao (1958-1978)

\begin{tabular}{|c|c|c|c|c|c|c|c|c|c|c|c|c|}
\hline \multirow[b]{2}{*}{ MES } & \multicolumn{11}{|c|}{ NUMERO DE AÑOS CON TEMPERATURAS MÍNIMAS $\leq 0$} & \multirow{2}{*}{$\begin{array}{l}\text { DÍAS } \\
\text { OBS. } \\
\text { (n) }\end{array}$} \\
\hline & $\leq 0$ & $\leq-1$ & $\leq-2$ & $\leq-3$ & $\leq-4$ & $\leq-5$ & $\leq-6$ & $\leq-7$ & $\leq-8$ & $\leq-9$ & $\leq-10$ & \\
\hline \multicolumn{13}{|c|}{ ENERO } \\
\hline $1-31$ & 0 & 0 & 0 & 0 & 0 & 0 & 0 & 0 & 0 & 0 & 0 & 649 \\
\hline \multicolumn{13}{|c|}{ FEBRERO } \\
\hline $1-28$ & $2(2)$ & 0 & 0 & 0 & 0 & 0 & 0 & 0 & 0 & 0 & 0 & 590 \\
\hline \multicolumn{13}{|c|}{ MARZO } \\
\hline $1-31$ & $3(4)$ & 1 & 0 & 0 & 0 & 0 & 0 & 0 & 0 & 0 & 0 & 650 \\
\hline \multicolumn{13}{|l|}{ ABRIL } \\
\hline $1-15$ & $1(1)$ & 0 & 0 & 0 & 0 & 0 & 0 & 0 & 0 & 0 & 0 & 630 \\
\hline $16-30$ & $8(24)$ & $3(9)$ & $1(2)$ & $1(1)$ & 0 & 0 & 0 & 0 & 0 & 0 & 0 & \\
\hline \multicolumn{13}{|l|}{ MAYO } \\
\hline $1-15$ & $13(51)$ & $11(28)$ & $7(14)$ & $2(3)$ & $1(2)$ & $1(1)$ & 0 & 0 & 0 & 0 & 0 & 651 \\
\hline $16-31$ & 19(99) & $16(74)$ & $11(36)$ & $10(19)$ & $3(5)$ & $2(3)$ & $1(2)$ & 0 & 0 & 0 & 0 & \\
\hline \multicolumn{13}{|l|}{ JUNIO } \\
\hline $1-15$ & $21(143)$ & $19(95)$ & $17(48)$ & $13(27)$ & $8(14)$ & $2(4)$ & 0 & 0 & 0 & 0 & 0 & 630 \\
\hline $16-30$ & $21(212)$ & $21(180)$ & $20(143)$ & $18(82)$ & $14(44)$ & $9(17)$ & $5((8)$ & $1(1)$ & 0 & 0 & 0 & \\
\hline
\end{tabular}




\begin{tabular}{|c|c|c|c|c|c|c|c|c|c|c|c|c|}
\hline \multicolumn{13}{|c|}{ JULIO } \\
\hline $1-15$ & $21(160)$ & $21(133)$ & $21(100)$ & $15(57)$ & $10(30)$ & $5(12)$ & $4(6)$ & 0 & 0 & 0 & 0 & 651 \\
\hline $16-31$ & $20(175)$ & $20(131)$ & $17(80)$ & $12(40)$ & $7(27)$ & $4(13)$ & $2(9)$ & $1(5)$ & $1(5)$ & $1(5)$ & $1(1)$ & \\
\hline \multicolumn{13}{|c|}{ AGOSTO } \\
\hline $1-15$ & 19(199) & $17(83)$ & $13(39)$ & $9(25)$ & $5(15)$ & $2(5)$ & $2(3)$ & 0 & 0 & 0 & 0 & 649 \\
\hline $16-31$ & $18(63)$ & $13(36)$ & $6(14)$ & $3(5)$ & $1(1)$ & 0 & 0 & 0 & 0 & 0 & 0 & \\
\hline \multicolumn{13}{|c|}{ SETIEMBRE } \\
\hline $1-15$ & $6(15)$ & $4(6)$ & $4(4)$ & 0 & 0 & 0 & 0 & 0 & 0 & 0 & 0 & 630 \\
\hline $16-30$ & $2(5)$ & 1(3) & $1(2)$ & 0 & 0 & 0 & 0 & 0 & 0 & 0 & 0 & \\
\hline \multicolumn{13}{|c|}{ OCTUBRE } \\
\hline $1-15$ & $5(5)$ & 1(1) & 0 & 0 & 0 & 0 & 0 & 0 & 0 & 0 & 0 & 651 \\
\hline $16-31$ & 0 & 0 & 0 & 0 & 0 & 0 & 0 & 0 & 0 & 0 & 0 & \\
\hline \multicolumn{13}{|c|}{ NOVIEMBRE } \\
\hline $1-30$ & $3(4)$ & $2(3)$ & $1(1)$ & 0 & 0 & 0 & 0 & 0 & 0 & 0 & 0 & 630 \\
\hline \multicolumn{13}{|c|}{ DICIEMBRE } \\
\hline $1-31$ & 1(2) & 0 & 0 & 0 & 0 & 0 & 0 & 0 & 0 & 0 & 0 & 651 \\
\hline
\end{tabular}

Fuente: Salazar (1979)

\section{Periodo vegetativo de los cultivos}

El tiempo que requiere un cultivo para producir depende de varios factores como la temperatura, agua, horas sol, etc.

La temperatura es la condición que determina la dirección del flujo neto de calor entre dos cuerpos (OMM, 2014) Esta magnitud permite expresar el nivel de temperatura de los cuerpos, en meteorología casi siempre la temperatura $(\mathrm{T})$ se mide en grados Celsius.

La FAO, UNESCO, OMM (1975) indican la siguiente variación en periodos vegetativos con altura para una variedad de papa mejorada y una variedad nativa (cuadro 3)

\section{Cuadro 3}

Variación de periodo vegetativo de papa en alturas variados

\begin{tabular}{lll}
\hline Variedad & Altitud $(\mathrm{msnm})$ & Periodo vegetativo (días) \\
\hline \multirow{3}{*}{ Antarqui } & Costa & 105 \\
& 3,000 & $100-110$ \\
& 4,000 & $120-130$ \\
Criolla & Costa & $150-180$ \\
& 3,000 & $150-200$ \\
& 4,000 & $200-240$ \\
\hline
\end{tabular}

En general las variedades de papa que se adaptan a zonas de costa y sierra, alargan su periodo vegetativo de 20 a 30 días en la sierra en comparación con la costa; investigaciones realizadas en ecuador indican que algunas variedades de cebada que se sembraron entre los 2,800 y los 3,200 msnm. Tuvieron un periodo vegetativo de 5.5 a 6 meses, a 3,600 msnm. Estas variedades alargaron su periodo vegetativo hasta los 8 meses.

Para dos estaciones bolivianas se indica que en una estación con temperatura media de $8.4^{\circ} \mathrm{C}$. el periodo vegetativo es de 210 a 240 días y en la otra estación con temperatura media de $14^{\circ} \mathrm{C}$. el periodo vegetativo es de 150 a 180 días en ambas estaciones hay muy poca diferencia en radiación diaria, la estación con temperatura de $8.4^{\circ} \mathrm{C}$. tuvo 31 días con heladas mientras la otra estación tuvo 10 días de heladas (Salazar, 1979)

En estudios realizados en la estación del INÍA santa Ana, así como en los proyectos de irrigación Orcotuna, Chupaca, Apata, Sincos y Yanacancha (Quispe, 1982) indican 
variaciones en periodo vegetativo (cuadro 4 y 5) con altura para algunos cultivos, siendo estas hasta de 30 días con relación al cultivo de la papa, para cultivo de cebada y trigo a alturas mayores de 3,500 msnm. Es de 40 a 70 días más de periodo vegetativo.

Cuando los cultivos se siembran en época de heladas (en terrenos bajo riego), el periodo vegetativo se alarga significativamente con relación a lo sembrado en época de lluvias (campaña grande), en la estación experimental Santa Ana del INIA Huancayo a $3,319 \mathrm{msnm}$. Se obtuvieron los siguientes resultados durante la campaña de noviembre a mayo y de noviembre a abril (con riego)

\section{Cuadro 4}

Periodo vegetativo de cultivos para 2 campañas al año en la Estación experimental Santa Ana Huancayo.

\begin{tabular}{|c|c|c|c|c|c|}
\hline \multirow{3}{*}{ Cultivo/variedad } & \multicolumn{5}{|c|}{ Periodo vegetativo (días) } \\
\hline & \multicolumn{3}{|c|}{$\begin{array}{l}\text { Campaña mayo } \\
\text { a noviembre }\end{array}$} & \multicolumn{2}{|c|}{$\begin{array}{l}\text { Campaña noviembre a } \\
\text { abril }\end{array}$} \\
\hline & 1979 & 1980 & 1981 & $1979 / 1980$ & $1980 / 1981$ \\
\hline \multicolumn{6}{|l|}{ Haba (en verde): } \\
\hline Pacae blanco & - & 152 & 165 & 140 & 142 \\
\hline Sinave & - & 132 & 152 & 129 & 136 \\
\hline Pacae verde & - & 132 & 165 & 140 & 144 \\
\hline \multicolumn{6}{|l|}{ Arveja (en verde) } \\
\hline Alderman & - & 133 & 135 & 125 & 123 \\
\hline Azul & - & 133 & 135 & 125 & 123 \\
\hline Criolla & - & 143 & 140 & 130 & 128 \\
\hline \multicolumn{6}{|l|}{ Trigo } \\
\hline Ollanta & 180 & 183 & 175 & 158 & 142 \\
\hline Cahuide & - & - & 175 & 158 & 142 \\
\hline $\mathrm{UNC}-2$ & 205 & 201 & 190 & 176 & 171 \\
\hline Huanca & 181 & 201 & 190 & 164 & 170 \\
\hline \multicolumn{6}{|l|}{ Cebada } \\
\hline J.A. Zapata & 177 & 165 & 165 & 141 & 144 \\
\hline \multicolumn{6}{|l|}{ Triticale } \\
\hline Mapache & 208 & - & - & 168 & - \\
\hline \multicolumn{6}{|l|}{ Avena } \\
\hline Mantaro 15 & 170 & - & - & 150 & - \\
\hline \multicolumn{6}{|l|}{ Hortalizas: } \\
\hline $\mathrm{Col}$ & 210 & 205 & - & 170 & 175 \\
\hline Lechuga & 128 & 130 & - & 120 & 112 \\
\hline Zanahoria & 164 & 160 & - & 150 & 146 \\
\hline cebolla & 165 & 170 & - & 150 & 152 \\
\hline
\end{tabular}

Fuente: (MINAG, 1982)

De igual modo en estudios realizados en diferentes lugares y en diferentes pisos ecológicos donde se ejecutaron proyectos de irrigación se obtuvieron los siguientes resultados (cuadro 5) 


\section{Cuadro 5}

Periodo vegetativo de cultivos en época de heladas y en diferentes pisos ecológicos (msnm), campaña de mayo a noviembre (en días)

\begin{tabular}{|c|c|c|c|c|c|}
\hline \multirow[b]{2}{*}{ Cultivo } & \multicolumn{5}{|c|}{ Proyectos de irrigación } \\
\hline & $\begin{array}{c}\text { La Huaycha } \\
(3330)\end{array}$ & $\begin{array}{c}\text { Chupaca } \\
(3313)\end{array}$ & $\begin{array}{l}\text { Apata } \\
(3300)\end{array}$ & $\begin{array}{c}\text { Chicche } \\
\text { (3700) }\end{array}$ & $\begin{array}{c}\text { Yanacancha } \\
(3900)\end{array}$ \\
\hline Haba (en verde) & 163 & 175 & 163 & 229 & 253 \\
\hline Arveja (en verde) & 154 & 164 & 155 & 230 & 250 \\
\hline \multicolumn{6}{|l|}{ Hortalizas: } \\
\hline $\mathrm{Col}$ & 185 & 180 & 185 & 230 & 230 \\
\hline Cebolla & 185 & 180 & 185 & 230 & 230 \\
\hline Zanahoria & 160 & 160 & 160 & 210 & 210 \\
\hline Lechuga & 122 & 120 & 125 & 150 & 150 \\
\hline
\end{tabular}

Fuente: (MINAG, 1982)

Se menciona que, en las zonas de Chicche y Yanacancha, las temperaturas mínimas en algunos días de los meses de mayo a julio llegaron a registrar $-5^{\circ} \mathrm{C}$ y a $-10^{\circ} \mathrm{C}$, afectando a los cultivos tanto en su crecimiento lento como en algunas partes de la misma planta (zona apical), finalmente dichos cultivos presentaron tolerancia al frio, prosiguiendo con su desarrollo normal en los meses posteriores.

\section{Frecuencia de riego}

La frecuencia de riego, entendido como la oportunidad de efectuar otro riego después de otro y de acuerdo a la necesidad hídrica del cultivo, para ello es necesario realizar una programación de riegos según la demanda del cultivo a fin de proveer del recurso hídrico en su oportunidad y en la calidad y cantidad necesaria.

En estudios realizados por el Ministerio de Agricultura, ensayos realizados para diferentes cultivos y en tipo de suelo franco arcillosa, con un control fitosanitario adecuado y todas las practicas agronómicas efectuadas se evaluaron los rendimientos obtenidos frente a la frecuencia de riego variados, con resultados que figuran en el cuadro siguiente.

\section{Cuadro 6}

Rendimiento total obtenido (kg/ ha) por cultivo frente a diferentes frecuencias de riego (FR) aplicado (días) en la campaña (mayo - noviembre)

\begin{tabular}{|c|c|c|c|c|c|c|}
\hline \multirow{2}{*}{ CULTIVO/VARIEDAD } & \multicolumn{2}{|c|}{1979} & \multicolumn{2}{|c|}{1980} & \multicolumn{2}{|c|}{1981} \\
\hline & $\begin{array}{c}\text { F.R. } \\
\text { (días) }\end{array}$ & $\begin{array}{c}\text { Rdto. } \\
\text { (kg/ha) }\end{array}$ & $\begin{array}{l}\text { F.R. } \\
\text { (días) }\end{array}$ & $\begin{array}{c}\text { Rdto. } \\
\text { (kg/ha) }\end{array}$ & $\begin{array}{c}\text { F.R. } \\
\text { (días) }\end{array}$ & $\begin{array}{c}\text { Rdto. } \\
\text { (kg/ha) }\end{array}$ \\
\hline \multicolumn{7}{|l|}{ HABA } \\
\hline Pacae blanco (en verde) & - & - & 14 & 13,409 & 15 & 10,234 \\
\hline Pacae verde (en verde) & - & - & 14 & 8,419 & 15 & 7,500 \\
\hline Sinave (en verde) & - & - & 14 & 4,304 & 15 & 7,000 \\
\hline \multicolumn{7}{|l|}{ ARVEJA } \\
\hline Alderman (verde) & - & - & 14 & 2,756 & 21 & 3,689 \\
\hline Azul (en verde) & - & - & 14 & 3,496 & 21 & 2,960 \\
\hline Criolla (en verde) & - & - & 14 & 2,304 & - & - \\
\hline \multicolumn{7}{|l|}{ TRIGO } \\
\hline Ollanta & 15 & 3,190 & 20 & 3,331 & 20 & 4,677 \\
\hline
\end{tabular}




\begin{tabular}{lcccccc}
\hline Huanca & 15 & 5,363 & 20 & 3,776 & 20 & 5,422 \\
UNC - & 15 & 3,941 & 20 & 3,518 & - & - \\
CEBADA & & & & & & \\
J.A. Zapata & 15 & 3,282 & 20 & 4,898 & 20 & 4,700 \\
TRITICALE & 15 & 3,800 & - & - & - & - \\
$\quad$ COL & & & & & & - \\
Corazón de buey & 7 & 1,800 doc. & 7 & 2,000 doc. & - & - \\
$\begin{array}{l}\text { Quintal } \\
\text { ZANAHORIA }\end{array}$ & 7 & 1,200 doc. & 7 & 1,800 doc. & - & - \\
Chantenay & 7 & 40,000 & 7 & 45,000 & - & - \\
LECHUGA & & & & & & - \\
Boston White & 7 & 12,000 doc. & 7 & 11,500 doc. & - & - \\
\hline
\end{tabular}

Fuente: (MINAG, 1982)

De este cuadro se deduce que los cultivos de haba y arveja obtienen buenos rendimientos con frecuencia de 14 a 15 días, los cultivos de trigo y cebada obtienen buenos rendimientos con frecuencias de 15 a 20 días y las hortalizas responden satisfactoriamente a frecuencias de riego de cada 7 días, entendiéndose estas frecuencias de riego para suelos de textura franco-arcillosa, dichos resultados variaran según el cambio de textura de suelo.

Planificación de la doble campaña de producción

Teniendo en consideración los resultados de investigación realizados en la Estación experimental Santa Ana y los proyectos de irrigación, así como la bibliografía consultada podemos sugerir algunas estrategias para realizar una planificación de la doble compaña de producción, es decir obtener dos cosechas en los doce meses del año, siguiendo el siguiente procedimiento general.

- Temperaturas medias mensuales, en lo posible obtener información local de temperaturas medias en forma mensual de las estaciones meteorológicas existentes en los lugares más cercanos al lugar donde se planificará la doble campaña agrícola durante los doce meses del año.

- $\quad$ Temperaturas mínimas mensuales, Es muy necesario este dato a fin de hacer el análisis de información de las bajas temperaturas que ocasionan las heladas en los meses críticos de los cultivos tolerantes a las heladas, esta información facilitara una elaboración optima de una cedula de cultivos, considerando los cultivos en campaña grande con lluvias y la campaña chica con riego y las bajas temperaturas.

- Información climática adicional, de acuerdo al método utilizado para calcular la demanda de agua del cultivo se obtienen datos de humedad relativa, radiación solar, horas de sol u otra información necesaria, y en caso especial obtener datos históricos de precipitación para el número mayor de años para ser utilizado en el balance hídrico a efectuarse.

- Disponibilidad de agua para riego, esto se debe obtener de datos históricos de aforos en los canales, ríos, quebradas o fuentes de agua de riego y/o de otros análisis hidrológicos. 
- Periodo vegetativo de cultivos adaptables a la zona, cultivos normalmente sembrados durante época de heladas, algunas veces los agricultores practican la doble campaña de producción con cultivos que suelen denominar "cultivos primerizos" y conocen los posibles cultivos y la frecuencia de éxito o fracaso.

- Considerar los mercados, se determina cuando son las épocas de mayor escasez del producto en los mercados, la posibilidad para desarrollar nuevos mercados y la rentabilidad de los cultivos en las diversas fechas.

- Determinar el peligro de las heladas, de acuerdo a un análisis detallado de frecuencia e intensidad de bajas temperaturas que ocasionan las heladas, se determina el peligro para los diversos cultivos posibles en la zona durante todo el año, considerando básicamente los "periodos críticos" de dichos cultivos.

- Determinar posibles fechas de siembra y cosecha, se determina los cultivos posibles a considerar para la siembra tolerantes a las bajas temperaturas y para la época bajo lluvia; es preciso considerar para ello las temperaturas medias, tolerancia a las heladas en las etapas de desarrollo del cultivo, periodo vegetativo de los cultivos posibles en la zona, así como la intensidad de lluvias y duración de la misma a fin de no afectar la maduración de algunos cultivos o dificultar la preparación del terreno para la nueva siembra.

- Determinar cedula tipo para la zona, esto se hace de acuerdo a ciertas consideraciones como la capacidad de uso de la tierra, las fechas posibles de siembra y cosecha de los cultivos, la rotación de cultivos deseada, tiempo requerido para la preparación de terreno, susceptibilidad de fracaso de los cultivos por falta de agua y disponibilidad oportuna de mercados, se propone en el cuadro 7 , una cedula tipo para la doble campaña de producción.

\section{Cuadro 7}

Cedula de cultivo propuesto para la zona andina del Perú.

\begin{tabular}{|l|l|l|l|l|l|l|l|l|l|l|l|l|l|}
\hline \multirow{2}{*}{ Cultivo } & \multicolumn{9}{|c|}{ Mes } & \multicolumn{1}{l|}{ Cultivo } \\
\hline & E & F & M & A & M & J & J & A & S & O & N & D & \\
\hline PAPA & & & & & & & & & & & & Haba en verde \\
\hline MAIZ & & & & & & & & & & & & Arveja en verde \\
\hline HABA & & & & & & & & & & & & Trigo \\
\hline ARVEJA & & & & & & & & & & & & Cebada \\
\hline ZANAHORIA & & & & & & & & & & & & Haba en verde \\
\hline COL & & & & & & & & & & & & & Arveja en verde \\
\hline CEBOLLA & & & & & & & & & & & & Zanahoria \\
\hline APIO & & & & & & & & & & & & Lechuga \\
\hline AVENA & & & & & & & & & & & & Haba en verde \\
\hline TRIGO & & & & & & & & & & & & Arveja en verde \\
\hline CEBADA & & & & & & & & & & & & & Col o cebolla \\
\hline
\end{tabular}

Campaña grande con lluvias

Campaña chica en época de heladas y bajo riego

Fuente: elaboración propia 
- Determinar la cantidad de hectáreas a irrigar, durante los meses del año y de acuerdo a un balance hídrico, se hace con la finalidad de regular la distribución de agua para que los cultivos no carezcan de agua en los meses más críticos.

- Considerar los coeficientes de uso consuntivo (kc), el uso consuntivo que es definido como la cantidad total de agua utilizada por un cultivo en una determinada área más la evaporación de humedad del suelo o precipitación interceptada por la superficie de los cultivos y luego evaporada; en el presente artículo el coeficiente de uso consuntivo de un cultivo expresa la relación entre la demanda de agua de este cultivo mantenido a niveles óptimos de agua y la demanda ETA, es la evapotranspiración potencial del cultivo de referencia (Rye Grass)

En los cuadros 8 y 9, se puede apreciar los resultados obtenidos de coeficiente kc, en 3 cultivos para la zona andina tanto en época de heladas como en la época de lluvias.

\section{Cuadro 8}

Coeficiente de cultivo kec, a varias etapas de crecimiento en el valle del Mantaro hasta el 100\% de cobertura efectiva.

\begin{tabular}{lllllllllll}
\hline & \multicolumn{10}{c}{ Días desde la siembra hasta cobertura efectiva (\%) } \\
\cline { 2 - 11 } Cultivo & $\mathbf{1 0}$ & $\mathbf{2 0}$ & $\mathbf{3 0}$ & $\mathbf{4 0}$ & $\mathbf{5 0}$ & $\mathbf{6 0}$ & $\mathbf{7 0}$ & $\mathbf{8 0}$ & $\mathbf{9 0}$ & $\mathbf{1 0 0}$ \\
\hline Trigo & .19 & .25 & .38 & .48 & .68 & .88 & 1.0 & 1.1 & 1.13 & 1.15 \\
Papa & .10 & .12 & .18 & .24 & .32 & .52 & .75 & 1.04 & 1.35 & 1.45 \\
Lechuga & .11 & .12 & .16 & .29 & .38 & .45 & .56 & .72 & .98 & 1.13 \\
\hline
\end{tabular}

Fuente: (Quispe, 1981)

\section{Cuadro 9}

Coeficiente de cultivo kec, a varias etapas de crecimiento en el valle del Mantaro después del 100\% de cobertura efectiva.

\begin{tabular}{lllllllll}
\hline & \multicolumn{7}{c}{ Días después de cobertura efectiva (\%) } \\
\cline { 2 - 9 } Cultivo & $\mathbf{1 0}$ & $\mathbf{2 0}$ & $\mathbf{3 0}$ & $\mathbf{4 0}$ & $\mathbf{5 0}$ & $\mathbf{6 0}$ & $\mathbf{7 0}$ & $\mathbf{8 0}$ \\
\hline Trigo & 1.15 & 1.10 & 0.91 & 0.70 & 0.40 & 0.21 & 0.12 & 0.12 \\
Papa & 1.45 & 1.25 & 0.85 & 0.45 & 0.30 & 0.20 & 0.15 & 0.10 \\
Lechuga & 1.13 & 0.90 & 0.80 & 0.80 & 0.80 & 0.80 & 0.80 & 0.80 \\
\hline
\end{tabular}

Fuente (Quispe, 1981)

\section{CONCLUSIONES}

Se han presentado diversos resultados obtenidos en los diferentes experimentos realizados en la Estación experimental de Santa Ana del Instituto de investigación e innovación agraria así como en los proyectos de riego ejecutados por el Plan de mejoramiento de riego en la sierra MERIS I, siendo la principal conclusión de este documento sobre la posibilidad de la doble campaña de producción en un año se puede realizar con siembras programadas con cultivos tolerantes a las bajas temperaturas, cultivos precoces y de buen rendimiento con un mercado asegurado.

Se tienen algunas conclusiones generales: 
- Los cultivos ideales para la siembra en la época de bajas temperaturas (heladas) son: trigo, cebada, avena, centeno, triticale, haba, arveja y las hortalizas (col, zanahoria, cebolla, lechuga, beterraga, rabanito, etc.).

- Los cultivos ideales para la siembra en la campaña grande (con lluvias) son: papa, maíz, haba, arveja, trigo, cebada y hortalizas (cuadro 7)

- Se sobre entiende que en estas zonas donde se practique la doble campaña de producción, se debe realizar un buen manejo de suelos e incorporación de materia orgánica.

- Las frecuencias de riego adecuadas para un tipo de suelo franco-arcilloso y obtener los máximos rendimientos son: para haba y arveja cada 15 días, trigo y cebada cada 20 días y hortalizas cada 7 días.

- Se considerarán los datos presentados de uso consuntivo kc, para calcular la demanda de agua de los cultivos propuestos que fueron determinados por el método lisimétrico, a fin de disponer el recurso hídrico en forma programada y oportuna.

- A fin de realizar una efectiva labor de transferencia de tecnología hacia los agricultores, beneficiarios de los proyectos de riego, será necesario efectuar capacitación previa a los técnicos encargados de dicha labor, la misma que debe cumplirse mediante la utilización de diferentes métodos de extensión como las metodologías adecuadas de capacitación.

- Las zonas interandinas de nuestro país que oscilan entre los $2500 \mathrm{msnm}$. a alturas hasta de $3500 \mathrm{msnm}$. Inclusive, ofrecen buenas posibilidades de desarrollar cultivos alternativos de exportación con doble cosecha anual con la seguridad de contar con el recurso hídrico y un mercado asegurado con buenos precios y favorables para los agricultores.

- Los trabajos de investigación cuyos resultados positivos sirven para que el productor agrícola tenga la oportunidad de mejorar sus ingresos con la doble campaña de producción con un mercado seguro que generara mayores ingresos para sus familias y con ello mejorar su calidad de vida, la investigación es importante en estas zonas la que debería plantearse y ejecutarse por las entidades de investigación correspondiente incluido las universidades que juegan un papel importante en el desarrollo económico social de nuestro País, la que debe hacerse en forma integral.

- El papel del estado es fundamental en la transferencia de conocimiento y apoyo con la asistencia técnica y capacitación, asegurando la producción y el mercado, con metodologías adecuadas como la aplicación de las "Escuelas de campo" y el principio de "aprender haciendo" que fortalecerán las capacidades de nuestros agricultores de estas zonas. 


\section{REFERENCIAS BIBLIOGRÁFICAS}

FAO., UNESCO., OMM., (1975) Estudio agro climatológico de la zona andina (informe técnico) por M. Freire; J. Rea y J.Q. Rijks. Proyecto interinstitucional en agrometeorología, Roma.

FAO Organizacion de las Naciones Unidas para la Agricultura y la alimentacion. (2011a). El estado de los recursos de tierras y aguas del mundo para la alimentacion y la agricultura. La gestion de los sistemas en situacion de riesgo. Londres/Roma.

Garay Canales, O. B. (2009) manual de uso consuntivo del agua para los principales cultivos de los andes centrales peruanos. Manual, 1 - 30

Ministerio de agricultura, (1982) Avances de investigacion y estrategias para la doble campaña de produccion, Proyecto Especial de Pequeñas y Medianas Irrigaciones, convenio PEPMI - INIPA, Plan de mejoramiento de riego en la sierra, MERIS I, Huancayo.

Organizacion Meteorologica Mundial, O (2014). Guia de instrumentos y metodos de observacion meteorologicas - OMM - N 8, Tiempo - Clima - Agua. Suiza: OMM.

Quispe R.J. y Garay C.O., (1981) Informe técnico anual 1979-1981 Estación experimental agropecuaria Santa Ana, Convenio: Programa nacional de pequeñas y medianas irrigaciones PEPMI y el Instituto nacional de investigación y promoción agropecuaria INIPA, Huancayo.

Rodriguez Jimenez, Rosa Maria; Benito Capa, Agueda; Portela Lozano, Adelaida;. (2004). Meteorologia y climatologia, semana de la ciencia y la tecnologia. España: Fundacion española para la ciencia y la tecnologia.

Salazar Le Roy (1979) Guía para planificar la doble campaña de producción anual en la sierra del Perú, Dirección general ejecutiva del Programa nacional de pequeñas y medianas irrigaciones, Plan MERIS I, Lima.

Salazar Le Roy (1979) Guía para estudios de evapotranspiración e instalación de parcelas demostrativas con riego por superficie, Dirección General Ejecutiva del Programa Nacional de Pequeñas y Medianas Irrigaciones, Plan MERIS I, Lima.

MOPT (1992), Guías para la elaboración de estudios del medio físico (contenido y metodología). Ministerio de obras públicas, transporte y medio ambiente. España.

\section{CITAR COMO:}

Quispe Rodriguez, J., Quispe Quezada, U. R., \& Quispe Medrano, J. A. (2020). Estrategias para la doble campaña de producción en la Zona Andina del Perú. Puriq, 2(2), 68-80. https://doi.org/10.37073/puriq.2.2.66 